\title{
Commentary: The Year in Nuclear Receptor Control of Metabolism
}

\author{
David J. Mangelsdorf \\ Department of Pharmacology, Howard Hughes Medical Institute, University of Texas Southwestern \\ Medical Center at Dallas, Texas 75390-9050
}

\begin{abstract}
This commentary highlights some of the most important discoveries in the field of nuclear receptor control of metabolism that occurred over the past year (2009 to 2010). As might be expected in a field that encompasses several hundred active laboratories, the task of providing a balanced look at these discoveries was daunting. Thus, to help make the selection of these discoveries, a small panel of colleagues was recruited to help. After selecting the top candidate discoveries from a Google search and a PubMed search, the panel was asked to rank them. These final selections were presented at "The Year In Basic Science Session" of the Annual Meeting of the Endocrine Society, ENDO 2010, the highlights of which are reproduced in this article. (Molecular Endocrinology 24: 2075-2080, 2010)
\end{abstract}

$I_{\text {the }}^{\mathrm{n}}$ mammals there are 48 nuclear receptor genes, and they constitute the largest single family of transcription factors in vertebrates. Most of these receptors are ligand regulated. Nuclear receptors are commonly divided into three categories: the endocrine, adopted orphan, and true orphan receptors. The topics of all the articles highlighted this year focus on either adopted orphans or true orphans and include discoveries within the past year that address five groups of nuclear receptors.

The first group is the peroxisome proliferator-activated receptors (PPARs). In mammals, there are three types: $\operatorname{PPAR} \alpha, \operatorname{PPAR} \delta$, and $\operatorname{PPAR} \gamma$. The articles highlighting these receptors focused on $\operatorname{PPAR} \alpha$ and $\operatorname{PPAR} \gamma$, whose endogenous ligands are fatty acids. $\operatorname{PPAR} \alpha$ also binds to the fibrate class of drugs while PPAR $\gamma$ binds to the antidiabetic drugs, thiazolidinediones. The two receptors not only play important therapeutic roles in regulating metabolism, but they also act as physiologic regulators of starvation, fasting, and refeeding.

The second group of receptors we will discuss is the liver $\mathrm{X}$ receptors (LXRs), which bind oxysterols and are sensors for cholesterol. They have two primary actions. One is to regulate cholesterol homeostasis, and the other is to regulate

ISSN Print 0888-8809 ISSN Online 1944-9917

Printed in U.S.A.

Copyright (๑) 2010 by The Endocrine Society

doi: 10.1210/me.2010-0357 Received August 30, 2010. Accepted September 9, 2010

First Published Online September 29, 2010 inflammation. Two of the highlighted articles discuss novel results related to the roles of LXRs in cholesterol homeostasis and as antiinflammatory modulators.

The third group includes the orphan receptors Rev$\operatorname{erb} \alpha$ and Rev-erb $\beta$. The articles highlighted here specifically discuss work with Rev-erb $\alpha$. Rev-erb $\alpha$ is a recently adopted member of the nuclear receptor superfamily that is regulated by heme. In this presentation I will discuss a novel discovery about the role of Rev-erb $\alpha$ in the metabolism of heme.

The fourth group of receptors to be discussed is represented by the liver receptor homolog-1 (LRH-1), a true orphan receptor. Although phospholipids have been shown to bind LRH-1, it is not yet clear whether LRH-1 activity is regulated by phospholipids as ligands. LRH-1 is rather unique in that it has multiple roles, depending on where and when it is expressed during development and in adult animals. LRH-1 plays a role in the liver governing both metabolism and inflammation and it also plays a unique role during development.

The fifth group of receptors includes the estrogen receptor-related (ERR) receptors, and here I will focus on $\operatorname{ERR} \alpha$ in particular. Several years ago it was demon-

\footnotetext{
Abbreviations: APR, Acute phase response; ERR, estrogen receptor-related; FGF, fibroblast growth factor; Idol, inducible degrader of LDLR; LDL, low-density lipoprotein; LDLR, lowdensity lipoprotein receptor; $L X R$, liver $X$ receptor; NMR, nuclear magnetic resonance; PGC- $1 \alpha$, peroxisome proliferator-activated receptor $\gamma$ coactivator- $1 \alpha$; PPAR, peroxisome proliferator-activated receptor; SREBP, sterol-responsive element-binding protein; TZD, thiazolidinedione.
} 
strated that the ERRs can bind to synthetic steroids although their endogenous ligands have not been identified. $\operatorname{ERR} \alpha$ is a key regulator of energy metabolism.

\section{PPAR $\alpha$-inducted hepatokine FGF21 mediates adaptation to ketosis and starvation through the coactivator PGC-1 $\alpha$}

This discovery was published in two articles $(1,2)$. The first was a joint effort from the University of Texas Southwestern, from the laboratories of Shawn Burgess and Steve Kliewer/David Mangelsdorf. This first article is entitled "FGF21 Induces PGC- $1 \alpha$ and Regulates Carbohydrate and Fatty Acid Metabolism during the Adaptive Starvation Response." The second article is from Terry Maratos-Flier's laboratory at Harvard Medical School and it is entitled "Fibroblast Growth Factor 21-Deficient Mice Demonstrate Impaired Adaptation to Ketosis."

Previous work from both the Maratos-Flier laboratory and the groups at UT Southwestern had shown that fibroblast growth factor (FGF) 21 is a hormone secreted from the liver in response to starvation, a ketogenic diet, and fibrate drugs. Fibrate drugs work through $\operatorname{PPAR} \alpha$. Work from the two laboratories had also shown that FGF2 1 is a direct target of PPAR $\alpha$ and is responsible for many of the downstream effects that permit the body to adapt to fasting and starvation and to a ketogenic diet. These effects include increasing lipolysis from adipose stores. That lipid returns to the liver, where it is oxidized and used for ketogenesis and gluconeogenesis. FGF21 also plays a prominent role in energy conservation by decreasing physical activity and body temperature.

The two new articles from these laboratories were the first to report the phenotype of the FGF21-knockout mouse. All previous work in the FGF21 field has been done using gain-of-function studies. The new work demonstrated the physiological importance of these earlier effects. In addition, this work showed that the energy homeostatic coactivator, PGC- $1 \alpha$ (peroxisome proliferator-activated receptor $\gamma$ coactivator- $1 \alpha$ ), mediates many of the downstream hepatic effects of FGF21.

The UT Southwestern researchers worked with FGF21-knockout mice that had been fed or fasted. They used nuclear magnetic resonance (NMR) isotopomer analysis from perfused livers - that is, an ex vivo study from the livers of these mice. This analysis allowed them to directly measure and quantify a number of biological metabolic processes, including $\beta$-oxidation, ketogenesis, and gluconeogenesis. When wild-type animals were fasted, the researchers saw a robust activation of the three processes. What is remarkable about the FGF21-knockout mice is that they were completely refractory to this starvation or fasting response.
This study went on to show that FGF21 induces PGC- $1 \alpha$ as a means to drive many of these activities. During fasting, FGF21 stimulates the expression of PGC$1 \alpha$, which then regulates a number of target genes involved in gluconeogenesis and in fatty acid oxidation.

This regulation is absent in mice lacking FGF21. The Maratos-Flier researchers also demonstrated that PGC- $1 \alpha$ was no longer regulated in their FGF21-knockout animals. Furthermore, in mice lacking PGC- $1 \alpha$, the UT Southwestern researchers showed that PGC- $1 \alpha$ was required for many of the effects of FGF21.

Data from the Maratos-Flier study also showed the importance of FGF21 in mediating the metabolic effects of a ketogenetic diet. The most profound effect of such a diet should be weight loss, but this did not happen in the FGF21-knockout mice. Instead, they ate more and gained weight, and they developed severe hepatic-steatosis due to an inability to appropriately metabolize hepatic lipids.

\section{PPAR $\gamma$ ligands mediate osteoclastogenesis and bone loss through the coactivator PGC-1 $\beta$}

This discovery comes from the laboratory of Yihong Wong, an investigator at UT Southwestern. It is entitled "PGC- $1 \beta$ Mediates PPAR $\gamma$ Activation of Osteoclastogenesis and Rosiglitazone-Induced Bone Loss" (3).

The focus of this work was on the mechanism of action of rosiglitazone, a synthetic PPAR $\gamma$ ligand that is widely used as an antidiabetic drug. One of this drug's side effects is that it induces bone loss. That occurs because rosiglitazone enhances osteoclastogenesis and inhibits osteoclast-mediated bone formation.

In this study, the researchers identified the mechanism by which rosiglitazone enhances osteoclastogenesis and bone resorption. This occurs through a transcriptional network of three players: PPAR $\gamma, \operatorname{PGC}-1 \beta$, and ERR $\alpha$. PPAR $\gamma$ activation induces PGC- $1 \beta$ expression indirectly by down-regulating $\beta$-catenin and c-jun, which leads to an increase in PGC- $1 \beta$. PGC- $1 \beta$ then carries out two functions. The first is to act as a coactivator for PPAR $\gamma$, when activated by thiazolidinediones (TZDs), to drive c-fos induction. This leads to an increase in osteoclast differentiation. The second function of PGC- $1 \beta$ is to act as a coregulator of $\operatorname{ERR} \alpha$, which is also induced by TZDs. This drives the fatty acid oxidation/oxphos pathway and mitochondrial biogenesis and activation, which are necessary for osteoclast activity. The convergence of these pathways ultimately enhances osteoclast function.

The researchers identified both PGC- $1 \beta$ and ERR $\alpha$ as being important in this pathway, using both loss- and gain-of-function studies. Their data show that, when given TZDs, there was robust trabecular bone loss in wild-type mice but that this loss was essentially absent 
in knockout mice. Quantitation by histomorphometry showed that wild-type animals had an increase in both osteoclast number and the surface area where bone resorption occurs. Animals that are lacking either PGC-1 $\beta$ or ERR $\alpha$ were refractory to this activity. This discovery may have important implications for the design of new PPAR $\gamma$ agonists that might avoid these side effects.

\section{PPAR $\gamma$ ligands mediate their antidiabetic effects by blocking cdk5-induced phosphorylation of PPAR $\gamma$}

This discovery was made by Bruce Spiegelman's laboratory at the Dana Farber Cancer Institute at Harvard Medical School. The article reporting the discovery is entitled "Antidiabetic Drugs Inhibit Obesity-Linked Phosphorylation of PPAR $\gamma$ by Cdk5” (4).

Adipocytes and PPAR $\gamma$ are at the center of the insulinresistance/metabolic syndrome. PPAR $\gamma$ is required to store fat in adipose tissue. Activation by the TZD class of drugs not only leads to an increase in the storage of fat in adipocytes but also affects adipokine secretion. In particular, it up-regulates adiponectin and down-regulates antiinflammatory adipokines. That produces a number of actions in peripheral tissues. It decreases glucose output in the liver, increases glucose uptake in muscle, and decreases the infiltration of inflammatory macrophages into fat.

There are three paradoxes regarding PPAR $\gamma$ and insulin resistance. First, partial loss of function mutations in PPAR $\gamma$ in humans unambiguously cause severe insulin resistance. Second, PPAR $\gamma$ agonists improve insulin-resistance in diabetes, even though most PPAR $\gamma$ target genes are already fully "on" in obesity. Third, some PPAR $\gamma$ ligands with poor agonist activity still have marked antidiabetic actions. These findings have led to a conundrum about how these agonists might actually work. The answer to these puzzling observations may come from this discovery by Spiegelman's laboratory.

Normally in the adipocyte, when PPAR $\gamma$ is on, it drives the expression of the genes that lead to insulin sensitization. When high-fat or obese individuals get type 2 diabetes, inflammatory cytokines, lipids, and fatty acids signal to the adipocyte. That causes the activation of $\mathrm{cdk} 5$, which is a kinase that phosphorylates PPAR $\gamma$ at a unique site in its ligand-binding domain. The consequence of phosphorylating PPAR $\gamma$ is that doing so represses its activity in activating these genes. That leads to insulin resistance and ectopic lipid deposition. The researchers showed that the antidiabetic agonists may work by preventing PPAR $\gamma$ phosphorylation at that site. This action results in derepression of key target genes, leading to insulin sensitivity and decreased serum glucose output.
The importance of the phosphorylation status of $\operatorname{PPAR} \gamma$ was further highlighted in a study in humans in which eight newly diagnosed type 2 diabetics were treated for six months with $4 \mathrm{mg} / \mathrm{d}$ rosiglitazone. The patients were subjected to glucose clamp studies before and after treatment, as well as biopsies of their sc fat depots, where phosphorylation of PPAR $\gamma$ was measured. In seven of the eight patients, the researchers were able to show a change in phosphorylation status after treatment. That status correlated almost perfectly with increased glucose utilization and sensitivity in those patients. Taken together, this work provided the potential explanation for the disparate effects of PPAR $\gamma$ ligands, and it provides a new avenue for designing improved PPAR $\gamma$ drugs that might have fewer side effects and better efficacy.

\section{Nuclear oxysterol receptor LXR regulates cholesterol uptake by degrading the LDL receptor}

This discovery was made by Peter Tononoz's laboratory at the University of California at Los Angeles, and is entitled "LXR Regulates Cholesterol Uptake through Idol-Dependent Ubiquitination of the LDL Receptor" (5).

The cell has two main transcriptional mechanisms by which it senses and regulates cholesterol. The first is under low cellular cholesterol concentrations. In this situation, the cell is able to up-regulate the import of cholesterol and increase cellular cholesterol synthesis. This response is regulated by the transcription factor SREBP-2 (sterol-responsive element-binding protein-2), which senses low cholesterol levels and then activates cholesterol biosynthesis and increases the synthesis of low-density lipoprotein (LDL) receptor (LDLR) to bring cholesterol into the cell. Under high cholesterol concentrations, another sensing mechanism comes into effect. That mechanism is governed by LXR, which is activated by oxysterols in response to the high cholesterol load.

One of LXR's major target genes, which is expressed in response to high cellular cholesterol, encodes ABCA1 (a member of the ATP-binding cassette A transporters). ABCA1 transports cholesterol out of the cell. LXR also regulates a second pathway, which is the subject of this article. That pathway prevents cholesterol uptake into cells by decreasing LDLR concentrations. Tontonoz and his colleagues showed that LXR activity induces another direct target gene that encodes a protein called Idol (inducible degrader of LDLR). This article also showed that Idol is a specific E3 ubiquitin ligase that targets and degrades LDLR and other family members, which occurs in a variety of peripheral tissues. Interestingly, Idol does not appear to be regulated by LXR in the liver, which makes sense considering the liver is the major organ responsible for removing cholesterol from the body. 
Finally, the researchers showed in a gain-of-function study that if Idol is overexpressed in the liver using an adenovirus, it leads to degradation of LDLR. The authors went on to demonstrate that Idol is an LDLR familyspecific E3 ligase. Together, this work established the importance of the LXR pathway in maintaining cellular cholesterol homeostasis. It also identified Idol as a potential new player and therapeutic target.

\section{Orphan receptor LRH-1 can replace Oct4 for programming induced pluripotent stem cells}

The next article comes from the laboratory of Huck Hui Ng at the National University of Singapore, Department of Biochemistry, in the Stem Cell Biology Institute, and it is entitled "The Nuclear Receptor Nr5a2 Can Replace Oct4 in the Reprogramming of Murine Somatic Cells to Pluripotent Cells" (6).

$\mathrm{Nr} 5 \mathrm{a} 2$ is the gene code for LRH-1. LRH-1 plays a major role in metabolism in the liver, but it also has an interesting role in stem cell biology, as this article demonstrates. The researchers screened for nuclear receptors that can enhance the reprogramming of fibroblasts, using the four Yamanaka factors. Only four factors are required-Oct4, Sox2, Klf4, and Myc-to reprogram fibroblasts into pluripotent stem cells. The researchers showed that two nuclear receptors were able to enhance this activity: LRH-1 and the pregnane X receptor. The data showed that a number of nuclear receptors also can dramatically repress reprogramming of the activity of these receptors.

In the experiment reported in this article, the researchers showed that LRH-1 can replace Oct 4 but not Sox 2 or $\mathrm{Klf} 4$, as one of the factors required for reprogramming. Austin Cooney's laboratory had previously shown that LRH-1 activates Oct 4 expression. In this article, the researchers demonstrated that other targets are also activated and, together with the three known factors, cause the reprogramming event that makes pluripotent stem cells. This is the first identification of a transcription factor that can replace Oct4 for reprogramming. This work also has another unique aspect. It shows that nuclear receptors have an interesting function in reprogramming. Finally, it suggests that there may be multiple ways of generating pluripotent stem cells.

\section{SUMOylation of LRH-1 and LXR $\beta$ mediates their antiinflammatory actions during the hepatic acute phase response (APR)}

The next article comes from Eckardt Treuter's laboratory at the Karolinska Institut in Stockholm. It is entitled "GPS2-Dependent Corepressor/SUMO Pathways Govern Anti-inflammatory Actions of LRH-1 and LXR $\beta$ in the Hepatic Acute Phase Response (7).
LRH-1 and the LXRs are known not only for their lipid metabolizing functions but also for their effects on inflammation. The work reported in this article shows that treatment with synthetic LXR or LRH-1 agonists induces specific SUMOylation (post-translational modifications of Small Ubiquitin-like Modifiers) of both LRH-1 and LXR $\beta$. The SUMOylation of these two proteins leads them to a transrepression pathway, which is mediated by recruiting the corepressor integrator protein GPS2. This protein allows these nuclear receptors, when they are SUMOylated, to associate with the N-CoR corepressor complex. When they are associated with the corepressor complex, the complex is stabilized and that leads to enhanced repression of inflammatory gene expression. During inflammation, one of the actions of the APR is to cause degradation of this corepressor complex. This in turn activates inflammatory gene expression by preventing the $\mathrm{N}-\mathrm{CoR}$ complex from binding to and inhibiting inflammatory gene promoters (e.g., NF- $\kappa \mathrm{B}$ ). LRH- 1 and LXR $\beta$ prevent degradation of the N-CoR complex, and this is one of the reasons why these receptors are able to repress inflammation.

Specifically, the researchers treated hepatocytes with inflammatory cytokines. This caused increased expression of the inflammatory marker, haptoglobin. Treatment with an LRH-1 or LXR agonist completely suppressed inflammatory cytokine induction of haptoglobin gene expression. This transrepression required SUMO-1 for the LRH-1-dependent activity and SUMO-2 and SUMO-3 for the LXR $\beta$-dependent activity. Knocking down expression of the SUMO proteins with siRNAs abolished the response to the two receptors' agonists. The dependence on SUMOylation of LRH-1 and LXR $\beta$ for this activity was shown biochemically in a chromatin immunoprecipitation experiment. In this experiment, recruitment of LRH-1 or LXR $\beta$ to the haptoglobin promoter was compromised when LRH-1 or LXR $\beta$ were mutated at their SUMOylation sites. In summary, this work further strengthened the role of ligand-dependent transrepression as an important mechanism for nuclear receptor action and it identified novel roles for both of these receptors in blocking the hepatic metabolic shutdown that occurs during the acute phase response.

\section{Orphan receptor Rev-erb $\alpha$ feedback regulates heme biosynthesis}

The next discovery comes from the laboratory of Mitchell Lazar at the University of Pennsylvania School of Medicine. The article reporting this discovery is entitled "Negative Feedback Maintenance of Heme Homeostasis by Its Receptor, Rev-erb $\alpha$ " (8).

Rev-erb $\alpha$ was first identified as a transcriptional repressor that mediates the negative limb of the circadian 
clock. It binds to genes that are activated by the RARrelated orphan receptors (ROR) $\alpha$ and $\gamma$, which are the positive regulators of the circadian clock. In 2005, Henry Krause's laboratory at the University of Toronto demonstrated that the Drosophila homolog of Rev-erb $\alpha$, a gene called E75, was a heme-binding receptor. In 2007, two independent groups, one from the laboratory of Mitchell Lazar and one from the laboratory of Tom Burris and Fraydoon Rastinejad, showed that Rev-erb $\alpha$, the human homolog, also binds heme. Those findings provided strong evidence that heme functions as a Rev-erb $\alpha$ ligand, which in turn enhances the repression activity of Reverb $\alpha$ by recruiting the co-repressor $\mathrm{N}-\mathrm{CoR}$.

Their recent article showed that overexpression of Rev-erb $\alpha$ causes a decrease in total cellular heme levels, whereas knocking out Rev-erb $\alpha$ expression causes an increase in heme levels. The principal components of this feedback loop for the receptor regulating its own ligand include both Rev-erb $\alpha$ and PGC- $1 \alpha$. When the demand for energy is high, PGC- $1 \alpha$ is active and this coordinately induces expression of metabolic genes and components of the circadian clock. Together this activity regulates expression of ALAS1, which is the rate-limiting enzyme in the production of heme. Heme is an important cofactor for oxidative mitochondrial enzymes that are also induced by the PGC- $1 \alpha$ pathway. As heme levels rise, they also bind to Rev-erb $\alpha$, which, in a coordinated feedback loop, represses the circadian clock, represses metabolic gene expression, and represses PGC- $1 \alpha$. This feedback loop in turn attenuates ALAS1 expression and lowers heme levels. The biological consequence of this feedback circuit is that it provides a way to maintain heme levels within a physiologic range that fluctuates according to nutrient status - and when more or less energy is needed.

\section{Drosophila orphan receptor DHR96 binds cholesterol and regulates cholesterol metabolism}

This discovery comes from Carl Thummel's laboratory at the University of Utah in collaboration with Henry Krause at the University of Toronto and is entitled "The Drosophila DHR96 Nuclear Receptor Binds Cholesterol and Regulates Cholesterol Homeostasis" (9).

An interesting aspect of nuclear receptor phylogenetics is the variable number of receptors present in different species. In humans there are 48 , half of which are ligands and half of which are orphans. Drosophila has 21 receptors; two of these have ligands, the rest are orphans.

The discovery by Thummel's group presents the case for a third liganded Drosophila orphan receptor, one that binds cholesterol-making this receptor pathway strikingly similar to the LXR pathway in humans. The authors purified the Drosophila orphan receptor DHR96 and showed that it copurifies with a peak that has identical mass to cholesterol. Furthermore, when the lipid fraction was extracted from the purified receptor protein and subjected to gas chromatography-mass spectrometry and an electron ionization spectrum, they discovered one major peak that comigrated perfectly with the mass of cholesterol and had its same spectral properties. There were also some minor peaks, and they were all sterols. These data strongly suggested that DHR96 can bind cholesterol. Though it is not yet clear whether cholesterol serves as a reversible ligand that can regulate DHR96 transcriptional activity similar to other nuclear receptors like LXR, this finding prompted the authors to explore the idea that this receptor may play a role in cholesterol homeostasis.

To that end, the researchers were able to show that flies, which unlike mammals are auxotrophs for cholesterol-meaning that they need to acquire cholesterol from their diet-required DHR96 to respond normally when fed a high-cholesterol diet. In DHR96 mutant flies a network of gene expression up- or down-regulated by cholesterol was dysregulated. In addition, DHR96-mutant flies were no longer able to take up cholesterol. Because these flies cannot repress excess cholesterol uptake, they accumulated lethal amounts of cholesterol.

The authors went on to show that one of the major regulators of this pathway is the protein NPC1B, which is the homolog of the mammalian protein NPC1L1, a target of the drug ezetimibe and an essential mediator of cholesterol absorption. When cholesterol concentrations are low, this protein is highly expressed to permit cholesterol uptake into the fly. When cholesterol accumulates in flies, expression of NPC1B is decreased to limit further uptake. This process is mediated by DHR96, and $n p c 1 b$ expression is dysregulated in DHR96-null flies. Under low cholesterol concentrations, DHR96 is permissive, allowing $n p c 1 b$ to be expressed and promoting cholesterol absorption; when cholesterol concentrations are high, DHR96 responds to cholesterol and in some way that is not yet clear represses NPC1B function, and this decreases cholesterol absorption. The authors went on to show that they can rescue the DHR96 null phenotype by suppressing the expression of $n p c 1 b$.

\section{C. elegans orphan receptor NHR-49 mediates adaptation to starvation and reproductive diapause}

This work was done in the laboratory of Marc Van Gilst at the Fred Hutchinson Cancer Research Institute in Seattle. It is entitled "Starvation Protects Germline Stem Cells and Extends Reproductive Longevity in C. elegans" (10).

Returning to the number of nuclear receptors in various species, the real winner is the nematode, C. elegans with 284 receptors. Only one of these receptors is known 
to have a ligand, although several of the others have been shown to have roles in regulating various metabolic processes. The discovery in this article characterizes the role of one of these orphan receptors as a metabolic regulator during fasting.

The receptor the authors investigated was NHR-49, the human homolog of $\mathrm{HNF} 4 \alpha$, a receptor known to be important in mammals in nutrient sensing, carbohydrate, and lipid metabolism. Previous work with Keith Yamamoto and Marc Van Gilst had suggested NHR-49 might have a similar role in regulating the fasted state.

In this article, NHR-49 targets were assessed by microarray analysis in both fed and fast states, and genes in the categories of fat and lipid metabolism were found to be targets, implicating a role in fat expenditure and lipid partitioning. In addition, the authors performed a two hybrid screen, and identified three other orphan nuclear receptors, NHR-80, NHR-13, and NHR-66, which evidently heterodimerize with NHR-49 to promote up-regulation of lipid synthesis and a repression of sphingolipid metabolism. These are key events required to survive a starvation response.

The authors went on to demonstrate that NHR-49 was required for worms to go into what is called adult reproductive diapause, which is essentially a starvation survival pathway. In response to three days of starvation, wildtype worms are able to survive while the NHR-49 mutants do not. The reason they survive is because they can arrest reproductive development, which is a very energydemanding process. The NHR-49 null animals are not able to arrest reproductive development, and as a consequence they rapidly deplete their energy reserves and die of starvation.

When the nematodes make the transition from a larval 4 stage to an adult and are ramping up the process of reproductive metabolism, they require a lot of energy that comes from their diet. During starvation, when that energy source is gone, NHR-49 responds by arresting reproductive development and activating a process of autophagy and phagocytosis that allows worms to use their own body as an energy source to survive starvation. When the animals start feeding again, the NHR-49 pathway turns off and the entire reproductive process restarts. A similar fasting and refeeding switch occurs in mammals, and it is regulated in part by HNF4, which is the mammalian homolog of NHR-49.

\section{Acknowledgments}

I thank all of the authors whose work improved our understanding of nuclear receptor control of metabolism in the past year: Steven Kliewer, Terry Maratos-Flier, Yihong Wan, Bruce Spiegelman, Peter Tontonoz, Eckardt Treuter, Huck Hui Ng, Mitch Lazar, Carl Thummel, and Marc Van Gilst.

Address all correspondence and requests for reprints to: David J. Mangelsdorf, University of Texas Southwestern Medical Center, Howard Hughes Medical Institute, Department of Pharmacology, 6001 Forest Park Road, Room ND9.124A, Dallas, TX 75390-9050. E-mail: davo.mango@utsouthwestern.edu.

Disclosure Summary: D.JM. is an SAB member at ReSet Therapeutics and at Aragon Pharmaceuticals, an Investigator of the Howard Hughes Medical Institute, is supported by the Robert A. Welch Foundation (I-1275), and serves in an advisory capacity for the Nuclear Receptor Signaling Atlas (NURSA).

\section{References}

1. Potthoff MJ, Inagaki T, Satapati S, Ding X, He T, Goetz R, Mohammadi M, Finck BN, Mangelsdorf DJ, Kliewer SA, Burgess SC 2009 FGF2 1 induces PGC- $1 \alpha$ and regulates carbohydrate and fatty acid metabolism during the adaptive starvation response. Proc Natl Acad Sci USA 106:10853-10858

2. Badman MK, Koester A, Flier JS, Kharitonenkov A, Maratos-Flier E 2009 Fibroblast growth factor 21-deficient mice demonstrate impaired adaptation to ketosis. Endocrinology 150:4931-4940

3. Wei W, Wang X, Yang M, Smith LC, Dechow PC, Wan Y 2010 PGC1 $\beta$ mediates PPAR $\gamma$ activation of osteoclastogenesis and rosiglitazone-induced bone loss. Cell Metab 11:503-516

4. Choi JH, Banks AS, Estall JL, Kajimura S, Boström P, Laznik D, Ruas, JL, Chalmers MJ, Kamenecka TM, Blüher M, Griffin PR, Spiegelman BM 2010 Anti-diabetic drugs inhibit obesity-linked phosphorylation of PPAR $\gamma$ by Cdk5. Nature 466:451-457

5. Zelcer N, Hong C, Boyadjian R, Tontonoz P 2009 LXR regulates cholesterol uptake through idol-dependent ubiquitination of the LDL receptor. Science 325:100-104

6. Heng J-CD, Feng B, Han J, Jiang J, Kraus P, Ng J-H, Orlov YL, Huss M, Yang L, Lufkin T, Lim B, Ng H-H 2010 The nuclear receptor $\mathrm{Nr} 5 \mathrm{a} 2$ can replace Oct4 in the reprogramming of murine somatic cells to pluripotent cells. Cell Stem Cell 6:167-174

7. Venteclef N, Jakobsson T, Ehrlund A, Damdimopoulos A, Mikkonen L, Ellis E, Nilsson L-M, Parini P, Janne OA, Gustafsson, JA, Steffensen KR, Treuter E 2010 GPS2-dependent corepressor/ SUMO pathways govern anti-inflammatory actions of LRH-1 and LXR $\beta$ in the hepatic acute phase response. Genes Dev 24:381-395

8. Su N, Yin L, Hanniman EA, Joshi S, Lazar MA 2009 Negative feedback maintenance of heme homeostasis by its receptor, Reverb $\alpha$. Genes Dev 23:2201-2209

9. Horner MA, Pardee K, Liu S, King-Jones K, Lajoie G, Edwards A, Krause HM, Thummel CS 2009 The Drosophila DHR96 nuclear receptor binds cholesterol and regulates cholesterol homeostasis. Genes Dev 23:2711-2716

10. Angelo G, Van Gilst MR 2009 Starvation protects germline stem cells and extends reproductive longevity in C. elegans. Science 326: 954-958 